\title{
Médiévales
}

Langues, Textes, Histoire

70 | printemps 2016

Lieux d'hygiène et lieux d'aisance en terre d'Islam (VII $\mathrm{e}_{-}$ $\mathrm{XV}^{\mathrm{e}}$ siècle)

\section{Sophie PAGE, Magic in the Cloister. Pious Motives, Illicit Interests and Occult Approaches to the Medieval Universe}

University Park (PA), The Pennsylvania State University Press, 2013, 232 p. (« Magic in History »)

\section{Nicolas Weill-Parot}

\section{OpenEdition}

\section{Journals}

Édition électronique

URL : http://journals.openedition.org/medievales/7755

DOI : 10.4000/medievales.7755

ISSN : $1777-5892$

\section{Éditeur}

Presses universitaires de Vincennes

Édition imprimée

Date de publication : 23 juin 2016

Pagination : 274-277

ISSN : 0751-2708

Référence électronique

Nicolas Weill-Parot, « Sophie PAGE, Magic in the Cloister. Pious Motives, Illicit Interests and Occult Approaches to the Medieval Universe », Médiévales [En ligne], 70 I printemps 2016, mis en ligne le 20 juillet 2016, consulté le 25 septembre 2020. URL : http://journals.openedition.org/medievales/7755 ; DOI : https://doi.org/10.4000/medievales.7755

Ce document a été généré automatiquement le 25 septembre 2020.

Tous droits réservés 


\section{Sophie PAGE, Magic in the Cloister. Pious Motives, Illicit Interests and Occult Approaches to the Medieval Universe}

University Park (PA), The Pennsylvania State University Press, 2013, 232 p. (« Magic in History »)

Nicolas Weill-Parot

\section{RÉFÉRENCE}

Sophie PAGE, Magic in the Cloister. Pious Motives, Illicit Interests and Occult Approaches to the Medieval Universe, University Park (PA), The Pennsylvania State University Press, 2013, 232 p. (« Magic in History »)

1 La magie médiévale a fait l'objet, surtout depuis les années 2000, d'un nombre assez important d'ouvrages et d'articles spécialisés. Les questions de la classification de traditions magiques, de leur possible survivance dans les normes de plus en plus strictes de la théologie à partir du XIII ${ }^{\mathrm{e}}$ siècle et du profil des utilisateurs sont délicates. Le livre de Sophie Page en partant d'une étude de cas, à la fois précise et révélatrice, apporte de très importants éléments de réponse.

2 Dans un premier chapitre, S. Page présente ce qui est la collection médiévale de textes de magie la plus abondante qui nous soit connue : celle de l'abbaye bénédictine anglaise Saint-Augustin. Ces ouvrages nous sont révélés à partir de la copie réalisée au $\mathrm{XV}^{\mathrm{e}}$ siècle d'un catalogue antérieur élaboré entre 1375 et 1420 , ainsi que par les témoins retrouvés. Cette richesse s'explique par les donations et les encouragements donnés aux moines pour se constituer des collections personnelles ensuite versées au monastère, l'absence de contrôle épiscopal sur le monastère, mais aussi la présence de moyens matériels pour réaliser des opérations magiques (pharmacie, ateliers 
métallurgiques, etc.). S. Page présente les différents donateurs, des moines venus avec leurs ouvrages qui sont allés ensuite à la bibliothèque du monastère : Adam (au début du XIII siècle), Guillaume de Clara (entré en 1277), Thomas de Sprot (ou de Willesborough), ordonné vers 1278 , et à qui le monastère doit l'entrée du très riche manuscrit Corpus Christi 125 ; Jean de Londres et Michael Nothgate, anciens étudiants de Paris, entrés tous deux dans les années 1320. La collection comporte des textes de différentes traditions magiques : magie "naturelle ", magie " des images " hermétique, salomonienne... En revanche elle ne compte pas d'ouvrages de nigromancie (c'est-àdire tournés vers la conjuration des démons, soit leur soumission à la volonté du magicien). Il n'en demeure pas moins que les textes de magie encouraient théoriquement, pour une grande partie d'entre eux, les foudres des théologiens orthodoxes. Leur abondante présence est donc une question que S. Page cerne tout au long du livre avec beaucoup de discernement. Dans les chapitres suivants, elle identifie chacune des traditions magiques qui émerge de l'étude de cette collection de manuscrits pour l'analyser en elle-même et dans ses rapports avec le milieu et les aspirations monastiques.

3 Le chapitre 2 est consacré à ce que S. Page regroupe sous l'expression de "magie naturelle». Elle donne à ce concept une certaine souplesse qui s'adapte aux données diverses, voire parfois contradictoires, des manuscrits étudiés. D'emblée, elle indique que cette notion recouvre trois aspects: descriptif (les propriétés occultes et autres procédés), explicatif (les raisons de leur efficience), et pratique (« instructions sur la manière de les utiliser »). Du point de vue théorique, elle rappelle l'importance de la réflexion de Guillaume d'Auvergne. La question du caractère "magique » de phénomènes naturels qui pourraient être qualifiés seulement de merveilleux est esquissée. S. Page propose deux critères susceptibles de se conformer au point de vue médiéval. Le premier est la présence d'un agent démoniaque : la présence du démon caractérisant la magie, mais dans ce cas, notons-le, la magie ne serait pas naturelle. Le second consisterait à considérer le but de l'opération - ce qui permettrait d'identifier les éléments magiques au sein de textes non magiques, en particulier médicaux. Peutêtre pourrait-on cependant suggérer que la question de l'artifice, de l'intervention humaine, aurait pu aussi être retenue pour distinguer le merveilleux naturel, à savoir sans intervention de l'homme, de la magie naturelle, à savoir la magie non "destinative" (sans adresse à quelque intelligence que ce soit, en particulier non démoniaque) ; la magie commençant lorsque l'homme intervient dans l'élaboration de l'objet (par exemple, un talisman, objet artificiel, à la différence de l'amulette, simple objet naturel utilisé tel quel). De manière à la fois pragmatique et claire, S. Page analyse ainsi les éléments de magie naturelle que l'on peut trouver dans les manuscrits de l'abbaye Saint-Augustin (volumes $\mathrm{n}^{\circ} 1267,1275$ et 1277 [Corpus Christi 125]). Elle montre l'importance de l'utilisation des parties de l'animal, un thème qui s'accorde avec les prescriptions de la Genèse (I : 28) offrant à l'homme la domination sur les animaux. Comme elle le remarque, Albert le Grand, avec les textes apocryphes qui lui sont attribués, est l'une des autorités les plus présentes. L'usage des membres d'animaux a souvent des buts concernant la sexualité, ce qui n'est pas nécessairement surprenant dans un milieu religieux, puisque, comme elle le rappelle, dans ses œuvres authentiques, Albert le Grand a décrit des contraceptifs et des abortifs alors même que, théologiquement, il condamnait l'une et l'autre pratique.

4 S. Page montre de manière convaincante que la magie naturelle des textes conservés pouvait être insérée dans une perspective de restauration de l'équilibre entre l'homme 
et la nature - équilibre perdu depuis la Chute et ce, de trois façons : (1) par l'acquisition d'une connaissance tirée de l'observation des animaux eux-mêmes (ainsi, le fait que l'hirondelle soigne ses petits avec du jus de chélidoine), (2) par l'amélioration de la capacité d'élevage (ainsi, se faire obéir des vaches), (3) par l'évocation du jardin d'Éden en permettant à l'homme de dominer les bêtes sauvages (un signe de sainteté). Enfin, s'il était admis que toute magie naturelle ou démoniaque ne pouvait dépasser les limites du cadre naturel (le privilège surnaturel étant réservé au miracle divin), les autorités de l'Église pouvaient trouver à redire aux pratiques de la magie naturelle même si elles étaient dépourvues d'invocations explicites adressées aux esprits - en cinq points que S. Page relève : "l'utilisation d'objets naturels moralement douteux ", l'assimilation par l'homme de qualités animales, l'utilisation de corps naturels pour contrôler des esprits, la création d'êtres vivants, et la métamorphose d'hommes en animaux.

5 Le chapitre 3 est consacré à l'étrange Liber vaccae. L'abbaye en conservait une copie dans deux manuscrits, celle du manuscrit Corpus Christi 125 est quelque peu censurée. S. Page analyse ce texte assez précisément en montrant comment le premier livre en particulier est déroutant : il vise à créer des créatures rationnelles et irrationnelles en extrayant l'âme d'animaux. Ses procédures s'écartent de la magie naturelle habituelle et reposent notamment sur la manipulation de créatures vivantes. Il repose sur l'idée d'une «malléabilité de la matière » et sur le caractère "impressionnable » de l'âme humaine sous l'effet de fumigations. À juste titre, S. Page compare les processus décrits à ceux de l'alchimie. En de nombreux points, le texte pouvait heurter le dogme chrétien; Guillaume d'Auvergne considérait que le magicien agissant ainsi était comparable à un démon; mais le texte lui-même ne comportait pas de traits explicitement démoniaques.

6 Le chapitre 4 consacré à la magie des images s'intéresse en particulier aux textes contenus dans le ms. Corpus Christi 125. Ils relèveraient plutôt de la dite tradition " hermétique » que de la tradition « salomonienne » ou nigromantique : Liber imaginum lunae, De viginti quatuor horis, De imaginibus diei et noctis, De quattuor imaginibus magnis (tous trois attribués à Belenus), De discretione operis differencia ex iudiciis Hermetis, De quindecim stellis, ainsi que le court texte que S. Page met en lumière Glossulae super Librum imaginum (et qu'elle édite en annexe). Tout en constatant le caractère très peu théorique de ces textes (qui contraste avec la teneur philosophique d'un De radiis d'alKindī), S. Page essaie de saisir le caractère ambigu de l'appel à Dieu, considéré comme ultime garant de tout pouvoir (dans une perspective monothéiste) et la fonction de l'astrologie (présentée souvent comme un moyen de justification "scientifique »). Elle scrute aussi les éléments les plus contestables d'un point de vue théologique: le complexe statut des esprits invoqués, la présence de «characteres " - ces « mystérieux signes graphiques " souvent présents -, ainsi que les rituels mis en œuvre. À défaut d'une improbable théorie cohérente et globale, S. Page propose de faire émerger des "structures sous-jacentes et reliées»: la «correspondance entre les parties de l'opération » et « le transfert du pouvoir des formes supérieures aux inférieures ».

$7 \mathrm{Au}$ chapitre 5, S. Page s'attarde sur un ouvrage peu diffusé, dont elle fournit une traduction latine en annexe (après en avoir donné ailleurs une édition critique) : le Liber de essentia spirituum. Elle en souligne les deux traits originaux : le mariage entre une cosmologie néoplatonicienne (fondée sur des sources disponibles en latin : le Timée de Platon, Apulée...) avec la magie talismanique arabe, et le statut de son auteur 
anonyme qui assume pour lui-même une révélation directe et contemporaine à Séville au XIII ${ }^{\text {e }}$ siècle (au lieu de la revendication habituelle d'une antique filiation d'un savoir révélé à des autorités comme Hermès ou Salomon). Sa magie est fondée sur l'appel aux esprits régissant les planètes pour qu'ils envoient des esprits inférieurs, lesquels sont ensuite enfermés dans des talismans. Ce texte ne semble avoir été lu que par Guillaume d'Auvergne qui le condamne non seulement parce qu'à ses yeux ces esprits - au statut certes ambigu - ne peuvent être que des démons, mais aussi - et S. Page insiste sur ce point - parce que cette revendication d'un statut prophétique de la part de l'auteur lui apparaissait comme étant particulièrement inadmissible.

8 Le dernier chapitre analyse l'Ars notoria, cet art théurgique censé conférer la connaissance, qui a récemment fait l'objet d'études précises, notamment de la part de Julien Véronèse. Le statut des arts le situe aux marges de la magie et S. Page montre comment les prières, les notae et le désir de connaissance et de salut pouvaient, jusqu'à un certain point, rencontrer des pratiques et des aspirations du monachisme lui-même. Cette proximité explique le nombre relativement important de manuscrits d'Ars notoria conservés, dont deux se trouvent dans la bibliothèque de Saint-Augustin, malgré son caractère suspect voire condamnable aux yeux des autorités de l'Église.

S. Page se clôt par l'étude de l'utilisation faite au Xvi ${ }^{e}$ siècle par John Dee des manuscrits contenant des textes magiques provenant de cette bibliothèque dont il fit l'acquisition - une analyse fondée notamment sur ses annotations. Elle montre comment Dee pouvait tirer ces textes vers ses propres intérêts : l'attraction d'esprits dans des gemmes, l'invocation d'esprits pour l'aider dans ses opérations magiques, et l'accomplissement de rituels pour parvenir à une vision et acquérir ainsi la sagesse.

Ce résumé fort incomplet suffira sans doute à faire comprendre que le livre de S. Page permet des progrès considérables dans la connaissance de la magie médiévale et de son contexte culturel. Richard Kieckhefer avait parlé des utilisateurs de textes de magie rituelle nigromantique comme des membres d'un « clerical underworld ». S. Page, grâce à ce témoignage exceptionnel par son ampleur et sa cohérence des fonds manuscrits de l'abbaye de Saint-Augustin, nous montre de façon subtile et nuancée la complexité des rapports entre la spiritualité monastique orthodoxe et les différentes formes de magie qu'elle identifie. Comme elle le souligne, le monastère permit d'abriter de tels manuscrits, en raison notamment de la confiance accordée aux donateurs des livres et à la piété des moines eux-mêmes, en raison aussi du contexte codicologique et culturel qui, jusqu'à un certain point, établissait des ponts spirituels entre le domaine spirituel orthodoxe et la nébuleuse des idées et pratiques magiques (comme cela est visible pour l'ars notoria). Les études sur la magie médiévale devront désormais tenir grand compte des résultats significatifs de ce travail important. 